\title{
CLINICAL PRACTICE
}

\section{Health implications and management of women with opioid use disorder}

\author{
Lisa M. Hachey ${ }^{* 1}$, Jason A. Gregg ${ }^{1}$, Tamara L. Pavlik-Maus ${ }^{1}$, Jill S. Jones ${ }^{2}$ \\ ${ }^{1}$ College of Nursing, University of Cincinnati, Cincinnati, OH, United States \\ ${ }^{2}$ St. Elizabeth Physicians Group, Alcohol \& Drug Treatment Center, Falmouth, Kentucky, United States
}

Received: January 6, 2017

DOI: $10.5430 /$ jnep.v7n8p57
Accepted: March 6, 2017

Online Published: March 9, 2017

URL: https://doi.org/10.5430/jnep.v7n8p57

\begin{abstract}
Opioid use disorder has risen to epidemic proportions in the United States at an alarming rate in the past decade and is considered a leading public health concern. Women have a higher rate of acute and chronic pain conditions and are more likely to be prescribed opioids for pain management. The disproportionate incidence of opioid use, misuse, and progression to heroin and injectable drug use among reproductive age women is associated with increased morbidity and mortality. Of particular concern are the unique health risks opioid-dependent women face including immune system alterations, endocrinopathies, diminished fertility, psychosocial isolation, interpersonal violence, and unintentional overdose. Opioid use in pregnancy is associated with negative maternal and neonatal consequences and requires comprehensive, multidisciplinary services for the co-occurring medical, mental health, infectious disease, social stressors, and legal issues. Neonatal abstinence syndrome is linked to a cluster of physiological withdrawal symptoms and considered the primary adverse outcome of opioid exposure in newborns. Maternal medication-assisted treatment with methadone or buprenorphine to decrease the negative effects of neonatal withdrawal is the standard of care for opioid use disorders in pregnancy. The complexity of services required for maternal opioid use disorders requires collaborative and multidisciplinary management strategies to optimize maternal and neonatal outcomes.
\end{abstract}

Key Words: Opioid use disorder, Health consequences, Pregnancy, Methadone, Buprenorphine, Neonatal abstinence syndrome

\section{INTRODUCTION}

The United States has ranked highest for per capita opioid use in the past decade, consuming $80 \%$ of the world's opioid drugs, with an associated rise in opioid misuse, addiction use disorders, fatal overdoses, and heroin use. ${ }^{[1,2]}$ Opioid use is considered a global epidemic with the dramatic increase largely driven by countries with high socio-economic status; consumption nearly tripling since 1990 . Emerging evidence suggests the increase in consumption of prescription opioids is the driving force behind the escalating opioid epidemic in the United States, contributing to the associated rise in morbidity and mortality. ${ }^{[3]}$ Parallel to the general population, the sharp rise of opioid prescription use has increased in women of childbearing age, including pregnant women. The incidence of prenatal opioid use is 5.6 per 1,000 live births ${ }^{[4]}$ and approximately $85 \%$ of pregnancies in women with an opioid abuse disorder are unintended. ${ }^{[5]}$

From 2008 to 2012, nearly 40\% of Medicaid-insured and $28 \%$ of private-insured women filled at least one outpatient opioid prescription each year. Approximately $22 \%$ of pregnant women enrolled in Medicaid filled an opioid prescription, while $2.5 \%$ filled an opioid prescription for a quantity

\footnotetext{
*Correspondence: Lisa M. Hachey; Email: hacheylm@ucmail.uc.edu; Address: College of Nursing, University of Cincinnati, Cincinnati, OH, United States. 
greater than a 30 day supply. ${ }^{[6]}$ Women now represent a large percentage of prescription opioid abusers resulting in a female admission rate for opioid addiction treatment that has outnumbered male admissions in all categories. ${ }^{[2,7]}$ In part due to increased national attention towards the reduction of inappropriate opioid prescribing, coupled with the availability and affordability of heroin, the opioid epidemic has translated into heroin being the drug of choice for pregnant women. The largest increase of heroin use between 2002 through 2013 was among women, while two out of three pregnant women receiving medication-assisted opioid addiction treatment report a history of heroin use. ${ }^{[6]}$ Given the high rate of opioid use in women, healthcare practitioners require knowledge of gender specific differences for opioid use disorder, health consequences, medication-assisted treatment, and evidence-based management options; including pregnancy associated issues.

\section{Case presentation}

L.T. is a 21-year-old Caucasian woman who presented for an initial obstetrical visit at approximately 26 weeks gestation. She reported a positive home pregnancy test a few months ago, but did not seek care because the identity of the father was unknown. Additionally, she was undecided about continuing the pregnancy. Currently she is staying with a friend due to a recent eviction from her apartment. Her parents live in the area, but she does not maintain a relationship with them. During her initial visit L.T. appeared anxious with hand tremors and an inability to maintain eye contact. When verbally screened for substance use she admitted using up to $300 \mathrm{mg}$ of injected heroin daily for the past 18 months and hydrocodone (Vicodin) when available. She frequently shared needles and equipment with others. She disclosed a history of exchanging sex for drugs, money, and living arrangements. Her last heroin use was within 24 hours. Her past history of prescription opioid use began at 17 years of age following a right knee injury while playing soccer in high school. Her pain was managed with hydrocodone for approximately six months before and after a right meniscus surgical repair. After the prescription was discontinued, she sought illicit sources of hydrocodone for the next 18 months and eventually transitioned to heroin due to cost. L.T. disclosed smoking a half-pack of cigarettes per day and marijuana four to five times per week. Approximately one year ago she had an unsuccessful attempt to discontinue drug use on her own. Her past medical history is significant for a spontaneous abortion eight months ago at 10 weeks gestation. On exam, L.T. measured approximately 24 weeks with fetal heart tones present. All four extremities have old tracks and scars with more recent track marks on her neck. She agreed to a urine drug screen during intake which was positive for cannabis and opioids.

\section{GENDER-SPECIFIC DIFFERENCES FOR OPIOID ABUSE IN WOMEN}

The initial injury and exposure to opioids represented in the case presentation depicts a common path of opioid abuse and addiction for women. In this scenario an adolescent athlete became addicted following medical management of an injury and subsequently sought illicit drug sources to meet her new addiction. As healthcare providers, we must recognize potential risk factors in women that can lead to opioid abuse or addiction. Relative to men, gender-specific issues may influence women's exposure to and misuse of prescription opioids. Studies have shown the prevalence, intensity, and experience of pain in women differs in comparison to men. ${ }^{[8]}$ Some evidence suggest these gender-specific differences may be attributed to the increased incidence of chronic conditions such as musculoskeletal and inflammatory disorders, migraine headaches, interstitial cystitis, irritable bowel syndrome, and fibromyalgia in women. ${ }^{\left[{ }^{8,9]}\right.}$ Femalespecific factors including sex hormones, chronic pelvic pain, dyspareunia, and menstrual pain may also contribute to the differences in gender pain experiences. ${ }^{[10]}$

Medications with abuse potential are more frequently prescribed for women than men, while women report prescription opioids as their drug of choice. ${ }^{[1]}$ Opioid prescriptions rates for women across the life-span have increased over the past two decades, with approximately $8 \%-9 \%$ of women aged 65 and older using long-term opioid therapy for non-cancer pain management. ${ }^{[12]}$ Studies conducted in Canada and the United States have found women were $48 \%$ more likely to use prescription medications, including opioid prescriptions for pain management. ${ }^{[11,12]}$ In a large multisite study $(\mathrm{N}=29,906)$ conducted in the United States on gender-specific differences in the misuse of prescription opioids, women tended to be a younger age (21-34), less ethnically diverse, report more family conflicts, less likely to have a history of incarcerations, and less likely to be employed in skilled manual labor. ${ }^{[7]}$ Additionally, women were more prone to seek medical attention for pain and report a higher incidence of lifetime psychological problems including emotional, physical, and sexual abuse than male counterparts. ${ }^{[7,10]}$ Women have a higher incidence of trauma, including gender-based and intimate partner violence and subsequently experience the associated physical and psychological effects of trauma. Trauma survivors often present with acute and/or chronic physical health problems, anxiety, depression, and post-traumatic stress disorder (PTSD), which contribute to a greater risk for prescription opioid 
misuse. ${ }^{[10,13]}$ The association between trauma, chronic pain, mental health disorders, and prescription opioid use have been well documented in the literature.

These complex comorbid risk factors, opioid prescribing patterns, and gender differences in absorption and metabolism of opioid medications may contribute to the escalation of opioid misuse and subsequent adverse effects in women. ${ }^{[10]}$ The greatest risk factor for opioid misuse in women is taking a prescription opioid medication for pain. ${ }^{[7,10]}$ Compared to men, women typically obtain prescription opioid medication from family, friends, acquaintances, or providers; whereas men more frequently acquire opioids from dealers. ${ }^{[7]}$

\section{HeAlth implications OF OPIOId USE DISORDER IN WOMEN}

\subsection{Physical health consequences}

Studies have shown prescription and illicit opioid use is linked to adverse health consequences with an increase in morbidity and mortality. Common presenting complaints related to opioid use in women include headaches, opioidinduced bowel dysfunction, constipation, pelvic pain, menstrual disorders, and sexually transmitted infections (STIs). Women treated for headaches with opioid therapy may experience medication overuse headaches with subsequent chronicity of episodic headaches. ${ }^{[14]}$ One study determined the incidence of headaches nearly doubled following opioid use with an increase in frequency noted as monthly exposure to opioids increased; demonstrating a pattern of headache progression. ${ }^{[14]}$

The physiological changes that occur in pregnancy normally alter the maternal immune system. A particular concern to obstetrical and women's health care providers is the profound effect of opioids on the immune and endocrine systems, further compromising both the woman and her fetus. Evidence suggests opioids have an inhibitory effect on the immune system and even greater immunosuppression noted with an increase in duration of use. ${ }^{[15]}$ With the alteration in the immune system response there is susceptibility to infections and autoimmune disorders, increasing the incidence of local and systemic infections. ${ }^{[15]}$ The immune modulating effects of various opioids affect the immune system differently and are dependent on the type of drug, the host, and duration of exposure factors. The exception is buprenorphine, which does not appear to have an effect on immune function and has a high safety profile for use in pregnancy. ${ }^{[16]}$

Findings from the National Prescription Opioid Misuse, Abuse, and Diversion (NOMAD) Study (N = 344) concluded $72.5 \%$ of nonmedical prescription opioid users reported a lifetime history of at least one common infectious disease. ${ }^{[17]}$ The typical route of transmission is through sexual contact or exchange of bodily fluids through the sharing of needles with an infected intravenous (IV) drug abuser. ${ }^{[17]}$ Drug-related sexually transmitted infections (STIs) include hepatitis (A, $\mathrm{B}$, and C), HIV, chlamydia trachomatis, gonorrhea, and treponema pallidum. The prevalence of HIV/AIDS (1.2 million in 2015) and HCV (3.9 million in 2015) is increased in drug abusers, especially those who inject drugs. ${ }^{[18]}$ Approximately $20 \%$ of HIV/AIDS and $75 \%$ of $\mathrm{HCV}$ is linked to intravenous drug abuse. ${ }^{[17]}$

Of further interest is the link between opioid induced amenorrhea and opioid influence on sex steroid dysregulation and endocrine dysfunction. In a study of women ages 30-75 years $(\mathrm{n}=47)$, testosterone, estradiol, and dehydroepiandrosterone sulfate (DHEAS) levels were $48 \%$ to $57 \%$ lower in women with chronic opioid use and intact ovaries as compared to the control group $(p<.01-.05)$. Additionally, luteinizing hormone (LH) and follicle-stimulating hormone (FSH) levels were $30 \%$ lower in premenopausal and $70 \%$ lower in postmenopausal opioid users. ${ }^{[19]}$ The opioid-induced health risks for women include altered ovarian sex hormone production, infertility, hypogondotrophic hypogonadism, and potential for osteoporosis. ${ }^{[19]}$ Reproductive age women taking opioids have greater cumulative lifetime opioid exposure and associated endocrine, fertility, fetal, and neonatal health implications. ${ }^{[12]}$

\subsection{Psychological health consequences}

Co-occurring mental health disorders strongly predict and are associated with prescribed and non-medical use of opioids. ${ }^{[12]}$ Furthermore, those with a history of mental health disorders have a complexity of symptoms which increase the severity of their addiction. ${ }^{[20]}$ The vast majority of women in treatment for substance use disorder report prior traumatic life experiences with exposure to physical, emotional, and sexual abuse. ${ }^{[7]}$ In a study of opioid dependent pregnant women $(n=174)$, nearly $65 \%$ of the participants reported cooccurring mental health symptoms including anxiety (40\%), depression (32\%), mood disorders (49.6\%), and suicidal ideation (12.6\%) in the past 30 days. ${ }^{[20]}$ Additionally, the participants experienced family and social dysfunction, unemployment, and medical impairment. ${ }^{[20]}$ Often, these psychosocial dynamics persist and women experience a repetitive pattern of trauma, violence, and substance abuse. ${ }^{[7]}$ Early screening of women for a co-morbid history of PTSD and mood disorders, childhood abuse, and substance abuse allows for the coordination of multidisciplinary treatment interventions to address the complex needs of these women. 


\section{OPIOID DISORDER IN PREGNANCY}

The use of opioids in pregnancy has increased from 1.19 to 5.77 per 1,000 live births between 2000 through 2009, with a corresponding increase in neonatal abstinence syndrome (NAS) or withdrawal from drugs following birth. ${ }^{[4]}$ The management of the opioid-dependent woman during pregnancy poses additional challenges for practitioners due to the complexity and extensive multidisciplinary services required to care for the woman, fetus, and neonate during and after pregnancy. ${ }^{[21]}$ Substantial treatment barriers exist for opioiddependent women, including lack of gender-specific treatment facilities, social stigma, limited obstetric-addiction specialists, fear of legal repercussions, and childcare issues. ${ }^{[6]}$ State legislative efforts to penalize and prosecute women for assault for illegal narcotic use in pregnancy has increased recently, further discouraging women from seeking prenatal care and substance abuse treatment. ${ }^{[6]}$ Currently, 18 states mandate health care professionals to report suspected use of illicit substances during pregnancy. ${ }^{[22]}$

Recent trends in opioid use disorders indicate a diverse socioeconomic and demographic population of women have become addicted to heroin and enter into substance abuse treatment. ${ }^{[23]}$ However, women of color and low-income status disproportionately face greater barriers for appropriate treatment of substance abuse in pregnancy, which may contribute to the poor perinatal outcomes for this population. ${ }^{[21]} \mathrm{Ad}-$ verse outcomes associated with opioid use disorders include an increased risk for fetal growth restriction, placental abruption, fetal death, preterm labor, in utero meconium passage, and fetal distress. ${ }^{[24]}$ Women should be routinely screened for substance use and commended for seeking care. ${ }^{[6]}$ Verbal or written maternal screening should address previous or current use of alcohol, tobacco, prescription opioids, or illicit drugs. This is an opportunity to intervene and provide collaborative prenatal and substance abuse treatment under appropriate health care supervision to optimize maternal, fetal, and neonatal outcomes.

\section{NEONATAL ABSTINENCE SYNDROME}

The increased rate of neonatal abstinence syndrome has mirrored the escalating maternal opioid epidemic between 2000 through 2009, rising from 1.20 to 3.39 per 1,000 live hospital births and is associated with adverse neonatal outcomes. ${ }^{[4]}$ Health care expenditures related to opioid use in pregnancy have increased from 2000 and 2009, with an average neonatal admission cost of $\$ 39,400$ (2000) to $\$ 53,400$ (2009). ${ }^{[4]}$ The average length of stay is 16 days, with much of this time spent in the neonatal intensive care unit (NICU) for treating the pathophysiology of withdrawal..$^{[4,25]}$ Neonatal abstinence syndrome occurs shortly after birth with newborns exhibiting a cluster of central nervous system effects and withdrawal symptoms. ${ }^{[25]}$ Clinical presentations and behavioral features of opioid withdrawal involve central nervous system, gastrointestinal, and autonomic dysfunction, which may present differently for preterm neonates or those with polysubstance exposure. Of substantial concern is neonatal seizure activity, indicative of severe withdrawal and central nervous system involvement. Common withdrawal signs and symptoms may include 1) irritability 2) increased tone 3) high-pitched cry 4) vomiting 5) poor feeding and 6) temperature instability. ${ }^{[25]}$ It is difficult to determine the degree of fetal exposure and predict the severity of neonatal withdrawal symptoms due to the variability of illicit opioid sources and accurate interval, duration, and dosage of reported maternal opioid use. ${ }^{[25]}$ The standard of care for opioid use disorders in pregnant women is the initiation of a medication-assisted treatment program rather than opioid withdrawal. ${ }^{[6,24]}$

\section{Pharmacological management of ADDICTION DISORDER}

Currently, medication-assisted treatment strategies are recommended because they reduce fetal distress associated with opioid withdrawal. ${ }^{[6,26-28]}$ In addition, opioid detoxification and/or withdrawal has demonstrated decreased neonatal birthweight, greater risk of drug relapse, and increased risktaking behaviors such as intravenous drug use and criminal activities. $^{[6]}$ As such, three of the most commonly used medication-assisted treatment options for pregnant women include: methadone; buprenorphine; and buprenorphine plus naloxone. Of these, methadone remains the most commonly prescribed medication-assisted treatment option for pregnant women in large part due to the fact that it has been commercially available for more than 50 years resulting in it being the most studied strategy. ${ }^{[6,27,28]}$ Methadone treatment has demonstrated the following benefits 1) improved obstetrical care 2) reduced fetal mortality 3) improved fetal growth and 4) increased likelihood of being discharged home to the care of parents. ${ }^{[6,26,29]}$ Buprenorphine, an opioid agonist treatment option available since 2002, has demonstrated similar maternal outcomes to that of methadone; however, there are some additional fetal benefits to the use of buprenorphine in lieu of methadone 1) less fetal cardiac suppression 2) less fetal movement suppression 3) shorter treatment duration for neonatal abstinence syndrome and 4) shorter length of hospital stay. ${ }^{[6,27,28,30]}$

Buprenorphine plus naloxone, a combined opioid agonist and antagonist, is a newer medication-assist treatment option for opioid dependency that is now being prescribed to pregnant women. Although outcomes data is limited, it seems to demonstrate similar maternal outcomes to both methadone 
and buprenorphine alone. In regard to neonatal outcomes, the following findings are documented in comparison 1) higher average head circumference 2) shorter on average but remained within normal growth ranges and 3) lower Apgar scores at 5 minutes. Unlike Methadone, long-term data on neonatal outcomes using buprenorphine plus naloxone and buprenorphine alone is quite limited. ${ }^{[27]}$

In addition to outcomes data, medication-assisted treatment options are heavily influenced by policy. In several US locations, buprenorphine plus naloxone may be the only treatment option available to pregnant women. ${ }^{[27]}$ Currently, there are only 19 states that have substance abuse treatment programs tailored to pregnant women with 12 of those states giving pregnant women treatment priority. In addition, 20 states did not include methadone on the 2013 Medicaid preferred drug list, while all 50 states included buprenorphine on these lists as a preferred treatment option. ${ }^{[6]}$ Currently, there is not enough long-term safety data to recommend buprenorphine plus naloxone to pregnant women. If treatment access necessitates the use of buprenorphine plus naloxone, it is important for providers take extra precautions in monitoring fetal and neonatal outcomes. Otherwise, buprenorphine alone should be recommended because it has more established safety data available than buprenorphine plus naloxone. ${ }^{[27]}$

\section{Discussion}

Non-medical use of prescription opioids have escalated in the past decade to an unprecedented epidemic rate in the
United States. Women are disproportionately prescribed opioids more frequently and in larger amounts for acute and chronic pain, placing them at increased risks for adverse health effects and non-medical opioid abuse. Of particular concern are the health effects associated with opioid use in women, which include alterations in immune function and endocrinopathies. The non-medical use of opioids places a woman at risk for progression to heroin and polysubstance abuse, leading to co-morbidities that further compromise her health. Co-existing mental health disorders are prevalent with opioid use disorders, including a history of complex trauma stemming from violence, multiple forms of abuse, and high-risk behaviors. Pregnancy poses additional challenges in caring for women with opioid use disorders as the fetus is at risk for neonatal abstinence syndrome. Screening women for substance use and mental health disorders in the perinatal setting provides an opportunity to initiate a multidisciplinary, gender-specific care approach to optimize outcomes for mother and fetus. Medication-assisted treatment strategies are beneficial for the pregnant woman, increase prenatal care visits, and reduce neonatal withdrawal symptoms. The expansion of access to multidisciplinary comprehensive services for perinatal, substance abuse, and mental health treatment is essential as the demand rises in the midst of the current opioid epidemic.

\section{CONFLiCTS OF INTEREST Disclosure}

The authors declare that there is no conflict of interest.

\section{REFERENCES}

[1] Imtiaz S, et al. Harms of prescription opioid use in the United States. Substance Abuse Treatment, Prevention, and Policy. 2014; 9(43): 1-5. https://doi.org/10.1186/1747-597x-9-43

[2] Substance Abuse and Mental Health Services Administration, Behavioral health barometer: United States, 2015. Substance Abuse and Mental Health Services Administration: Rockville.

[3] Fischer B, et al. Correlations between population levels of prescription opioid use and prescription-opioid-related substance use treatment admissions in the USA and Canada since 2001. Public Health. 2012; 126(9): 749-51. PMid:22770736 https://doi.org/10.1 016/j.puhe .2012 .04 .010

[4] Patrick SW, et al. Neonatal abstinence syndrome and associated health care expenditures: United States, 2000-2009. JAMA. 2012; 307(18): 1934-40. PMid:22546608 https ://doi .org/10.1001/ jama.2012.3951

[5] Heil SH, et al. Unintended pregnancy in opioid-abusing women. Journal of Substance Abuse Treatment. 2011; 40(2): 199-202. PMid:21036512 https://doi.org/10.1016/j.jsat.2010.08 .011

[6] Krans E, Patrick S. Opioid use disorder in pregnancy: Health policy and practice in the midst of an epidemic. Obstetrics and Gynecology.
2016; 128(1): 4-10. PMid:27275812 https://doi.org/10.109 7/AOG.0000000000001446

[7] Green TC, et al. Women who abuse prescription opioids: Findings from the Addiction Severity Index-Multimedia Version Connect prescription opioid database. Drug and Alcohol Dependence. 2009; 103(1-2): 65-73. PMid:19409735 https ://doi.org/10.1016/j . drugalcdep. 2009.03.014

[8] Bartley EJ, Fillingim RB. Sex differences in pain: A brief review of clinical and experimental findings. British Journal of Anaesthesia. 2013; 111(1): 52-8. PMid:23794645 https://doi.org/10.109 $3 / \mathrm{bja} /$ aet 127

[9] Keogh E. Sex and gender differences in pain: A selective review of biological and psychological factors. Journal of Men's Health and Gender. 2006; 3(3): 236-243. https://doi.org/10.1016/j.jm hg. 2006.03.006

[10] Hemsing N, et al. Misuse of prescription opioid medication among women: A scoping review. Pain Research and Management. 2016. 1754195 p. PMid:27445597

[11] Simoni-Wastila L. The use of abusable prescription drugs: The role of gender. J Womens Health Gend Based Med. 2000; 9(3): 289-97. PMid:10787224 https://doi.org/10.1089/15246090031847 0 
[12] Darnall BD, Stacey BR, Chou R. Medical and psychological risks and consequences of long-term opioid therapy in women. Pain Medicine. 2012; 13(9): 1181-211. PMid:22905834 https://doi.org/10.1 $111 / j .1526-4637.2012 .01467 . x$

[13] Fareed A, et al. Comorbid posttraumatic stress disorder and opiate addiction: A literature review. Journal of Addictive Disease. 2013 32(2): 168-79. PMid:23815424 https://doi.org/10.1080/10 550887.2013 .795467

[14] Bigal ME, Lipton RB. Excessive opioid use and the development of chronic migraine. Pain. 2009; 142(3): 179-82. PMid:19232469 https://doi.org/10.1016/j.pain.2009.01.013

[15] Vallejo R, de Leon-Casasola O, Benyamin R. Opioid therapy and immunosuppression: A review. Am J Ther. 2004; 11(5): 354-65. PMid:15356431 https://doi.org/10.1097/01.mjt.0000132 250.95650 .85

[16] Al-Hashimi M, et al. Opioids and immune modulation: More questions than answers. British Journal of Anaesthesia. 2013; 111(1): 80-8. PMid:23794649 https ://doi .org/10.1093/bja/aet153

[17] Novak S, et al. The prevalence of common infectious diseases among nonmedical prescription opioid users: Preliminary findings from the National Prescription Opioid Misuse, Abuse, and Diversion (NOMAD) Study. Epidemiology. 2015; 5(4).

[18] Control CfD. HIV in the United states: At a glance 2015. 2015.

[19] Daniell HW. Opioid endocrinopathy in women consuming prescribed sustained-action opioids for control of nonmalignant pain. The Journal of Pain. 2008; 9(1): 28-36. PMid:17936076 https: //doi.org/10.1016/j.jpain.2007.08.005

[20] Benningfield MM, et al. Co-occurring psychiatric symptoms are associated with increased psychological, social, and medical impairment in opioid dependent pregnant women. American Journal of Addiction. 2010; 19(5): 416-21. PMid:20716304 https: //doi.org/10.1111/j.1521-0391.2010.00064.x

[21] Krans EE, Cochran G, Bogen DL. Caring for opioid-dependent pregnant women: prenatal and postpartum care considerations. Clinical Obstetrics and Gynecology. 2015; 58(2): 370-9. PMid:25775440 https://doi.org/10.1097/GRF.0000000000000098
[22] The Guttmacher Institute. Substance abuse during pregnancy. State policies in brief. 2015 [cited 2016 July 27]; Available from: https://www.guttmacher.org/print/state-policy/ explore/substance-abuse-during-pregnancy

[23] Thielke SM, et al. Age and sex trends in long-term opioid use in two large American health systems between 2000 and 2005. Pain Med. 2010; 11(2): 248-56. PMid:20002323 https://doi .org/10.111 $1 / j .1526-4637.2009 .00740 . x$

[24] American College of Obstetricians and Gynecologists, ACOG Committee Opinion No. 524: Opioid abuse, dependence, and addiction in pregnancy. Obstetric and Gynecology. 2012; 119(5): 1070-6. PMid:22525931 https://doi.org/10.1097/A0G.0b013e3182 $56496 \mathrm{e}$

[25] Sutter MB, Leeman L, Hsi A. Neonatal opioid withdrawal syndrome. Obstet Gynecol Clin North Am. 2014; 41(2): 317-34. PMid:24845493 https://doi.org/10.1016/j.ogc.2014.02. 010

[26] Minozzi S, et al. Maintenance agonist treatments for opiatedependent pregnant women. Cochrane Database Syst Rev. 2013; 12: Cd006318. https://doi.org/10.1002/14651858.cd006 318.pub3

[27] Lund I, et al. A comparison of buprenorphine + naloxone to buprenorphine and methadone in the treatment of opioid dependence during pregnancy: Maternal and neonatal outcomes. Substance Abuse: Research and Treatment. 2013; 2013(7): 61-74. PMid:23531704 https://doi.org/10.4137/SART.S10955

[28] Jones HE, Finnegan LP, Kaltenbach K. Methadone and buprenorphine for the management of opioid dependence in pregnancy. Drugs. 2012; 72(6): 747-57. PMid:22512363 https://doi .org/10. 216 5/11632820-000000000-00000

[29] Lund IO, et al. Prescription drug use among pregnant women in opioid Maintenance Treatment. Addiction. 2013; 108(2): 367-76. PMid:22882166 https : //doi .org/10.1111/j.1360-0443.20 12.04049.x

[30] Lacroix I, et al. Buprenorphine versus methadone in pregnant opioiddependent women: A prospective multicenter study. European Journal of Clinical Pharmacology. 2011; 67(10): 1053-9. PMid:21538146 https://doi.org/10.1007/s00228-011-1049-9 